\title{
Comportamento fenológico da palmeira patauá (Oenocarpus bataua) na reserva florestal Adolpho Ducke, Manaus, Amazonas, Brasil.
}

\author{
Roberto Rojas RUIZ ${ }^{1}$; Jurandyr da Cruz ALENCAR ${ }^{2}$
}

\begin{abstract}
RESUMO
Estudou-se a fenologia da palmeira patauá (Oenocarpus bataua Martius) na Reserva Florestal Adolpho Ducke em ecossistema de terra-firme ao longo de 16 anos, e calculou-se a ocorrência média mensal de dez fenofases. Usou-se a análise dos componentes principais entre dez variáveis fenológicas e seis meteorológicas. As porcentagens de ocorrência média mensal apresentaram valores baixos $(<14 \%)$ e ocorreram todo o ano. Pela análise dos componentes principais a precipitação e a insolação foram as variáveis abióticas que apresentaram alto poder discriminatório na formação da variação dos primeiros componentes, que juntos explicaram 89,6 \% da variação. As correlações entre a fenofase "cacho com frutos caindo" e as variáveis meteorológicas foram positivas com a insolação $(\mathrm{r}=0,34)$, temperatura máxima $(\mathrm{r}=0,31)$ e temperatura média $(\mathrm{r}=0,20)$, e negativa com a umidade relativa $(\mathrm{r}=0,-26)$ e precipitação $(\mathrm{r}=-0,26)$.
\end{abstract}

\section{PALAVRAS-CHAVE}

Fenologia, Floresta de Baixio, Palmeira, Amazônia Central.

\section{Phenology of the patauá palm (Oenocarpus bataua) in the Adolpho Ducke forest reserve, Manaus, Amazonas, Brazil.}

\begin{abstract}
Phenology of the Patauá palm (Oenocarpus bataua Martius) in the "terra-firme" ecossystem of the Adolpho Ducke Forest Reserve was studied for 16 years. Percentage average occurence of ten phenophases was calculated. Principal Component Analysis of ten phenological variables and six meteorological variables was done. Percentage average occurence of the phenophases presented low values $(<14 \%)$ occuring the whole year. Precipitation and insolation showed high discriminatory power in the formation of the first two principal components, as calculated by Principal Component Analysis, and together represented $89.6 \%$ of the total variation. Correlations were positive between "falling fruits" and insolation ( $r=0.34)$, maximum temperature $(r=0.31)$ and mean temperature $(r=0.20)$, and negative with precipitation $(r=-0,26)$ and relative bumidity $(r=-0.26)$.
\end{abstract}

\section{KEY WORDS}

Phenology, Swamp forest, Palm, Central Amazonia.

\section{INTRODUÇÃO}

Na Reserva Florestal Adolpho Ducke ocorreram 35 espécies em 14 gêneros de palmeiras, sendo 3 espécies do gênero Oenocarpus, (O. bacaba, O. bataua e O. minor) (Henderson \& Scariot, 1993). As palmeiras são fontes de alimentos para diversos animais, sendo o maior componente de suas dietas (Henderson et al., 1995). Das seis espécies denominadas "oligárquicas" na Amazônia, quatro são palmeiras (Euterpe oleracea, Oenocarpus bataua, Mauritia flexuosa e Orbignya phalerata), cujas populações naturais produzem até 11,1 toneladas de frutos/ha/ano, podendo gerar rendas substanciais e ecologicamente sustentáveis (Peters et al., 1989).

A fenologia é o estudo do comportamento dos vegetais em correlação a seu meio ambiente, abrangendo os padrões estacionais de floração, frutificação e folhação (Wood \& Burley, 1991; Bowers \& Dimmitt, 1994), tendo relevância para compreender o funcionamento dos ecossistemas naturais (Bullock \& Solis-Magallanes, 1990). IbarraManriquez (1992) relatou que estudos sobre a fenologia de palmeiras ao nível do Continente Americano são insuficientes para conhecer os padrões reprodutivos da família.Henderson (1995) e Kahn \& Granville (1992)

${ }^{1}$ Prof. Eng ${ }^{\mathrm{O}}$ Florestal M. Sc., Universidade Nacional da Amazônia Peruana, Faculdade de Engenharia Florestal. Rua Pevas 580, Iquitos, Peru.

${ }^{2}$ Prof. Eng ${ }^{\circ}$ Florestal, Dr., Instituto de Tecnologia da Amazônia UTAM/UEA, Curso de Engenharia Florestal. Rua Darcy Vargas, 1200, Manaus, AM, Brasil. 


\section{ACTA \\ AMAZONICA}

COMPORTAMENTO FENOLOGICO DA PALMEIRA PATAUA (Oenocarpus bataua) NA RESERVA FLORESTAL ADOLPHO DUCKE, MANAUS, AMAZONAS, BRASIL revisaram os poucos estudos que foram publicados, e concluíram que os períodos de floração e frutificação variaram na região amazônica, dependendo da estação seca ou úmida, e que o padrão fenológico foi supraanual. $\mathrm{Na}$ Guiana Francesa, a floração e frutificação de patauá foi bianual, com periodicidade regular (Sist, 1989 a). No Suriname, a floração ocorreu durante a estação chuvosa, e a frutificação de janeiro a abril (Wessels Boers, 1965). Segundo Ribeiro et al. (1999), o patauá floresceu nos meses de janeiro a novembro na Reserva Ducke. Na Colômbia, a floração ocorreu em períodos de pouca precipitação (Collazos \& Mejia, 1987). No Equador, observou-se que o patauá floresceu durante o ano todo, sendo que no fim da estação seca e início da estação chuvosa registrou-se um maior número de palmeiras em floração (García, 1988). Pedersen \& Balslev (1993) observaram que a floração de patauá não estava estritamente correlacionada com as mudanças climáticas. No Peru, no baixo Ucayali, a frutificação ocorreu o ano todo e os frutos maduros ocorreram com valor máximo (40\%) em setembro (Ruiz, 1985). Segundo Kahn (1990), neste mesmo local, a maior freqüência de frutos ocorreu durante a estação seca.

O presente estudo tem como objetivo analisar a fenologia da espécie Oenocarpus bataua Martius na Reserva Florestal Adolpho Ducke, correlacionando os dados fenológicos com valores de variáveis meteorológicas.

\section{MATERIAL E MÉTODOS}

Foram estudados 100 indivíduos com idade reprodutiva da espécie patauá, que crescem nas margens do igarapé Barro Branco, na Reserva Florestal Adolpho Ducke, no município de Manaus, Amazonas, Brasil, em ecossistema de terra-firme, área localizada em baixio, entre as coordenadas $59^{\circ} 52^{\prime} 40^{\prime \prime}$ e $59^{\circ} 58^{\prime} 00^{\prime \prime}$ de longitude Oeste e $03^{\circ} 00^{\prime} 00^{\prime}$ e $03^{\circ} 08^{\prime} 00^{\prime \prime}$ de latitude Sul (Alencar, 1994). As observações fenológicas foram feitas no período de agosto de 1978 a dezembro de 1994. Os dados meteorológicos foram obtidos na Estação Meteorológica da Reserva Ducke, gentilmente cedidos pela Coordenação de Pesquisas em Geociências do INPA. O clima é do tipo Afi, segundo a classificação de Köppen (Araújo, 1970).

A comunidade vegetal é dominada pela presença da palmeira arborescente patauá, com menor presença de açaí (Euterpe precatoria), muru-murú (Astrocaryum murumuru), paxiubinha (Iriartella setigera) e buriti (Mauritia flexuosa). As espécies de angiospermas arbóreas geralmente são de pequeno diâmetro, com raízes adaptadas a solos hidromórficos onde o lençol freático é muito superficial, sendo representadas por angelim (Hymenolobium beterocarpum), amapá (Brosimun sp.), breu-vermelho (Protium sp.), mulateiro (Peltogyne paniculata) e ucuúba-vermelha (Virola calophylla). Os solos são hidromórficos, inundados, com textura arenosa de cor cinza escuro, com lençol freático superficial, muito ácidos ( $\mathrm{pH} 4,11$ a 4,7), com teores de Fósforo (1 a 7 mg/ $\mathrm{dm}^{3}$ ), Potássio (8 a $\left.34 \mathrm{mg} / \mathrm{dm}^{3}\right)$; os teores de Cálcio e Magnésio são baixos (Rodriguez, 1995).

Para o estudo das fenofases adotou-se a metodologia de Alencar \& Anderson (1978), observando-se mensalmente o estado fenológico presente em cada cacho, cujas fenofases são: 01: Cacho com bainhas fechadas; 02: Cacho com botões expostos; 03: Cacho com flores abertas; 04: Cacho com flores caindo; 05: Cacho com flores caídas; 06: Cacho com frutos verdes; 07: Cacho com frutos maduros; 08: Cacho com frutos caindo; 09: Cacho com frutos caídos e 10: Palmeira sem cacho. As porcentagens médias mensais das fenofases foram calculadas pelo programa FENOPALM desenvolvido na CPST/ INPA sob a supervisão do $2^{\circ}$ autor. O cálculo da média mensal consistiu em dividir o número de ocorrências de cada fenofase, em cada mês para o período estudado, pelo número total de ocorrências, multiplicando-se em seguida por 100 . Trata-se pois, de transformar uma variável discreta numa variável quantitativa, expressa em porcentagem.

A análise dos componentes principais foi feita pelo programa STAT-ITCF (Philippeau, 1992) com os dados das médias mensais das 10 variáveis fenológicas e 6 variáveis meteorológicas (insolação, precipitação, temperatura máxima, temperatura média, temperatura mínima e umidade relativa), que gerou a matriz de correlações lineares, as porcentagens de contribuição das variáveis nos componentes principais e a representação plana das variáveis nos eixos, de acordo com Bouroche \& Saporta (1980) e Philippeau (1992).

\section{RESULTADOS E DISCUSSÃO}

\section{Clima}

A precipitação média anual, no período de 1978 a 1994, foi de 2401,5 mm e a precipitação média mensal foi de 200,1 $\mathrm{mm}$; os meses de junho a novembro apresentaram os menores índices de precipitação, abaixo de $200 \mathrm{~mm}$, sendo julho o mês de menor precipitação com 104,5 mm; os meses de dezembro a maio apresentaram as maiores precipitações, acima de $200 \mathrm{~mm}$, sendo janeiro e março os meses mais chuvosos, 276,4 e 274,6, respectivamente (Fig.1A). A temperatura média mensal no período variou entre $25,3^{\circ} \mathrm{C}$ e $26,3^{\circ} \mathrm{C}$; médias superiores a $26^{\circ} \mathrm{C}$ ocorreram de agosto a novembro, na estação seca. A temperatura máxima variou entre $30,8^{\circ} \mathrm{C}$ (janeiro) e $33,6^{\circ} \mathrm{C}$ (setembro), com os maiores valores entre agosto e novembro. A temperatura mínima apresentou valores entre $21,7^{\circ} \mathrm{C}$ (julho) e $22,5^{\circ} \mathrm{C}$ (março). A insolação média mensal variou entre 97,1 e 214,7 horas de brilho solar, em fevereiro e agosto, sendo que os meses de março a julho, novembro e dezembro tiveram valores menores que 200 horas; e em agosto observou-se o máximo valor (214,7 horas). O valor médio mensal da umidade relativa variou entre $82,6 \%$ (setembro) e $94,3 \%$ (fevereiro); os meses de agosto a novembro apresentaram os valores mais baixos, que correspondem à estação seca (estiagem). 


\section{ACTA AMAZONICA}

\section{Fenofases}

As porcentagens de ocorrência das fenofases foram baixas (Tab.1), variando de 1,9\% (frutos caindo) a $13,9 \%$ (cacho com bainha fechada). Estas porcentagens baixas podem ser causadas pelo moderado esforço reprodutivo desta palmeira, pois, segundo Balick \& Anderson (1986/ 87), somente $34,3 \%$ do total da produção de matéria seca acima do solo é destinado para as partes reprodutivas (19,6 $\%$ para as inflorescências e $14,7 \%$ para os frutos); o restante $65,7 \%$ é dirigido às folhas e ao estipe. As baixas

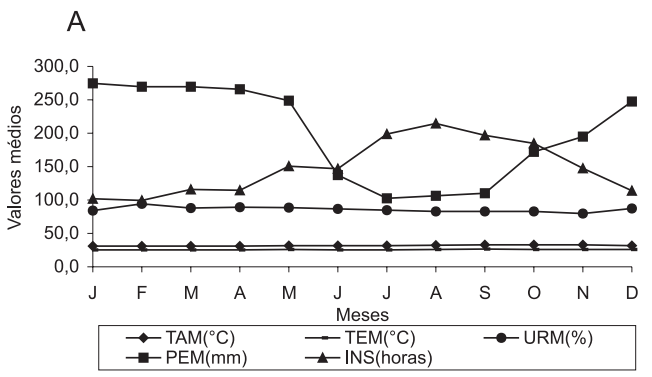

B

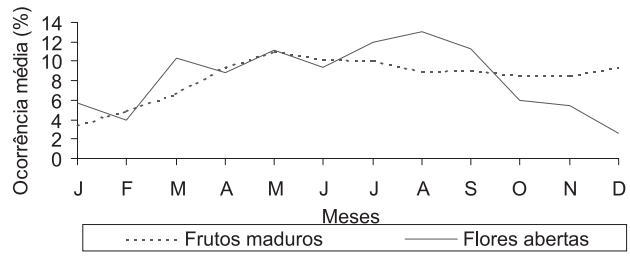

Figura 1 - Médias mensais das variáveis meteorológicas (A) e porcentagens médias mensais de flores abertas e frutos maduros (B) de patauá (Oenocarpus bataua Martius) no período de agosto de 1978 a dezembro de 1994 na Reserva Florestal Adolpho Ducke, Manaus, Amazonas, Brasil. porcentagens também podem estar relacionadas a fatores endógenos, pois a espécie apresentou uma grande variabilidade genética nas populações (Balick,1992).

A maior porcentagem na fenofase "cacho com bainhas fechadas" (13,9\% no mês de abril) pode ser explicada pelo fato de que quando a espécie começa a produzir inflorescências, a maioria das novas folhas tem um novo botão floral (Pedersen \& Balslev, 1993). Para as outras fenofases (02 a 09) as menores porcentagens (2,6 a 12,7\%) podem ser explicadas pela baixa eficiência reprodutiva desta espécie causada por pragas. No Equador, uma espécie de Derelomine, além de ser polinizadora, coloca ovos em 14\% a $63 \%$ das flores pistiladas e as larvas resultantes destroem o óvulo (García,1988). Alternativamente, acontecem abortos em face das deficiências de nutrientes nos solos de baixio (Rodriguez, 1995; Ferraz et al., 1998), onde os níveis de cátions trocáveis de $\mathrm{Ca}$ e $\mathrm{Mg}$ foram muito baixos.

No Equador, encontrou-se também que, de 65 botões florais, 14 morreram antes que estivessem formados os frutos maduros, com causa ainda desconhecida (Pedersen \& Balslev, 1993). Freqüentemente, ataques de Rynchophorum palmarun destroem as inflorescências que ainda estão fechadas nas suas bainhas (Collazos \& Mejia, 1987).

Entretanto, para a fenofase "palmeira sem cachos" as baixas porcentagens, em relação às outras fenofases, provavelmente podem estar relacionadas ao fato de que os cachos permanecem no caule por longo tempo, sendo difícil encontrar-se palmeiras adultas sem cachos.

Portanto, as baixas porcentagens de ocorrência média de patauá não podem ser explicadas somente pela influência das variáveis climáticas. Como se vê na matriz de correlações lineares (Tab.2), houve correlação positiva significativa entre a fenofase "cacho com frutos caindo" e as variáveis insolação, temperatura máxima e temperatura média; a fenofase "cacho com frutos caídos" apresentou correlações similares à fase

Tabela 1 - Porcentagem média mensal de ocorrência das fenofases de patauá (Oenocarpus bataua Martius), no período de agosto de 1978 a dezembro de 1994 na Reserva Florestal Adolpho Ducke, Manaus, Amazonas, Brasil.

\begin{tabular}{|c|c|c|c|c|c|c|c|c|c|c|c|c|}
\hline Fenofase & Jan & Fev & Mar & Abr & Mai & Jun & Jul & Ago & Set & Oct & Nov & Dez \\
\hline 01 & 7,1 & 6,2 & 10,2 & 13,9 & 12,7 & 9,3 & 8,4 & 7,7 & 6,2 & 6,4 & 6,8 & 5,1 \\
\hline 02 & 4,5 & 11,4 & 5,1 & 12,7 & 12,0 & 12,1 & 10,4 & 11,8 & 6,9 & 5,5 & 2,6 & 5,0 \\
\hline 03 & 5,7 & 4,0 & 10,3 & 8,9 & 11,1 & 9,4 & 12,0 & 13,1 & 11,3 & 6,0 & 5,5 & 2,6 \\
\hline 04 & 3,9 & 4,8 & 4,1 & 11,8 & 8,1 & 9,1 & 11,0 & 11,9 & 12,7 & 10,2 & 7,2 & 5,2 \\
\hline 05 & 6,2 & 4,4 & 4,8 & 9,9 & 10,1 & 6,4 & 8,3 & 9,3 & 10,6 & 11,7 & 10,6 & 7,6 \\
\hline 06 & 7,6 & 6,6 & 5,0 & 8,8 & 9,1 & 6,9 & 7,2 & 8,0 & 8,0 & 10,2 & 11,4 & 11,1 \\
\hline 07 & 3,4 & 4,9 & 6,6 & 9,4 & 11,0 & 10,2 & 10,0 & 9,0 & 9,1 & 8,6 & 8,5 & 9,4 \\
\hline 08 & 4,5 & 1,9 & 4,2 & 5,8 & 8,4 & 10,3 & 9,2 & 13,6 & 12,9 & 11,4 & 8,2 & 9,5 \\
\hline 09 & 6,7 & 6,0 & 6,6 & 5,6 & 6,1 & 6,8 & 7,2 & 9,2 & 11,3 & 12,4 & 11,4 & 10,7 \\
\hline 10 & 8,2 & 8,4 & 8,6 & 8,6 & 8,0 & 6,1 & 7,8 & 8,3 & 8,7 & 8,8 & 9,4 & 9,1 \\
\hline
\end{tabular}

Fenofase: 01- Cacho com bainhas fechadas; 02- Cacho com botões expostos; 03- Cacho com com com flores abertas; 04 - Cacho com flores caindo; 05 Cacho com flores caídas; 06- Cacho com frutos verdes; 07- Cacho com frutos maduros; 08- Cacho com frutos caindo; 09- Cacho com frutos caídos e 10Palmeira sem cacho. 
Tabela 2 - Matriz de correlações lineares das variáveis fenológicas de patauá (Oenocarpus ataua Martius) e meteorológicas da Reserva Florestal Adolpho Ducke, no período de agosto de 1978 a dezembro de 1994. Manaus, Amazonas, Brasil

\begin{tabular}{|c|c|c|c|c|c|c|c|c|c|c|c|c|c|c|c|c|}
\hline VAR & CBF & CBE & CFA & CFC & CFCA & CFV & CFM & CFFC & CFCC & PSC & INS & PEM & TAM & TEM & TIM & URM \\
\hline $\mathrm{CBF}$ & 1 & & & & & & & & & & & & & & & \\
\hline CBE & 0,54 & 1 & & & & & & & & & & & & & & \\
\hline CFA & 0,45 & 0,43 & 1 & & & & & & & & & & & & & \\
\hline CFC & 0,30 & 0,49 & 0,57 & 1 & & & & & & & & & & & & \\
\hline CFCA & 0,14 & 0,26 & 0,42 & 0,54 & 1 & & & & & & & & & & & \\
\hline CFV & 0,24 & 0,11 & 0,20 & 0,36 & 0,62 & 1 & & & & & & & & & & \\
\hline CFM & $-0,13$ & 0,01 & $-0,01$ & 0,14 & 0,21 & 0,32 & 1 & & & & & & & & & \\
\hline CFFC & $-0,20$ & $-0,15$ & $-0,14$ & $-0,08$ & 0,02 & 0,02 & 0,48 & 1 & & & & & & & & \\
\hline CFCC & 0,02 & $-0,11$ & $-0,11$ & $-0,10$ & $-0,17$ & $-0,14$ & $-0,02$ & 0,38 & 1 & & & & & & & \\
\hline PSC & 0,06 & $-0,06$ & $-0,09$ & $-0,19$ & $-0,23$ & $-0,25$ & $-0,25$ & $-0,22$ & 0,10 & 1 & & & & & & \\
\hline INS & $-0,06$ & 0,02 & 0,12 & 0,16 & 0,09 & 0,02 & 0,12 & 0,34 & 0,21 & 0,11 & 1 & & & & & \\
\hline PEM & 0,12 & 0,05 & $-0,01$ & $-0,01$ & $-0,01$ & 0,03 & 0,01 & $-0,26$ & $-0,13$ & 0,10 & $-0,65$ & 1 & & & & \\
\hline TAM & $-0,10$ & $-0,11$ & $-0,04$ & 0,10 & 0,10 & 0,12 & 0,12 & 0,31 & 0,25 & $-0,04$ & 0,63 & $-0,51$ & 1 & & & \\
\hline TEM & $-0,10$ & $-0,11$ & $-0,07$ & 0,06 & 0,13 & 0,09 & 0,12 & 0,20 & 0,003 & $-0,15$ & 0,20 & $-0,23$ & 0,58 & 1 & & \\
\hline TIM & 0,03 & $-0,13$ & $-0,17$ & $-0,05$ & 0,07 & 0,15 & $-0,08$ & $-0,09$ & $-0,05$ & $-0,05$ & $-0,18$ & 0,13 & 0,33 & 0,48 & 1 & \\
\hline URM & $-0,08$ & 0,07 & 0,01 & 0,01 & 0,02 & 0,04 & $-0,05$ & $-0,26$ & 0,24 & $-0,03$ & $-0,42$ & 0,32 & $-0,42$ & $-0,22$ & 0,03 & 1 \\
\hline
\end{tabular}

$\mathrm{CBF}=$ cacho com bainha fechada; $\mathrm{CBE}=$ cacho com botões expostos; $\mathrm{CFA}=$ cacho com flores abertas; $\mathrm{CFC}=\mathrm{cacho}$ com flores caindo; $\mathrm{CFCA}=\mathrm{cacho}$ com flores caídas, CFV = cacho com frutos verdes; CFM = cacho com frutos maduros; CFFC = cacho com frutos caindo; CFCC = cacho com frutos caídos; PSC = palmeira sem cacho; INS = insolação; PEM = precipitação; TAM = temperatura máxima; TEM = temperatura média; TIM = temperatura mínima; U RM = umidade relativa. $\mathrm{N}=204 ; \mathrm{gl}=202 ; \mathrm{p}=0,01 ; \mathrm{r}=0,182$ ( $\mathrm{N}$ eave, 1980 )

citada, exceto para a variável temperatura média; para estas duas fenofases as correlações com a umidade relativa foram negativas e significativas.

Pelo exposto, para explicar melhor este padrão fenológico, concorda-se com a hipótese formulada por Alencar (1994), de que: "os padrões fenológicos seriam mais afetados pelas condições endógenas das espécies (fisiológicas, nutricionais, edáficas, genéticas, modo de reprodução) e pelos vetores ecológicos (polinização, predação, competição) do que somente pela influência das variáveis climáticas."

A ocorrência das dez fenofases foi o ano todo; isto pode ser explicado pelo fato de que as inflorescências desta espécie têm um desenvolvimento muito prolongado. $\mathrm{O}$ tempo de desenvolvimento das inflorescências desde o aparecimento da bainha até a formação dos frutos maduros foi de cerca de dois anos no Equador (Pedersen \& Balslev 1993). Na Guiana Francesa, Sist $(1989$, b) estimou que a duração do amadurecimento dos frutos foi de \pm 16 meses, e os frutos maduros não disseminados persistiram vários meses na infrutescência.

Mostram-se ainda na Figura $1 \mathrm{~A}$ as curvas médias mensais das variáveis meteorológicas e na Figura $1 \mathrm{~B}$ as curvas de frutos maduros e flores abertas, por serem estas fenofases consideradas as de maior importância na floração e frutificação desta palmeira. Vê-se que a curva de frutos maduros cresce a partir de novembro, com máximo valor em maio, fim da estação chuvosa; a curva de flores abertas apresenta valores máximos entre julho e setembro, com pico em agosto, meses de maiores valores de insolação, que definem a estação seca.

\section{Correlações lineares entre as variáveis}

Na Tabela 2 observa-se que a fenofase "cacho com frutos caindo (08) apresentou correlação positiva significativa com a insolação, temperatura máxima e temperatura média, e correlação negativa significativa com a precipitação e umidade relativa. A fenofase "cacho com frutos caídos" (09) apresentou correlação positiva significativa com a insolação, temperatura máxima e com a umidade relativa. As outras fenofases não foram significativas com as variáveis meteorológicas.

\section{Análise dos Componentes Principais}

Os componentes principais 1 e 2 explicaram $89,6 \%$ da variação total e o restante da variação (10,4\%) referiu-se aos outros eixos. A redução de 16 variáveis originais ( 10 fenológicas e 6 meteorológicas) a duas variáveis não correlacionadas permitiu verificar que as variáveis precipitação e insolação foram as que tiveram maior contribuição na formação dos Componentes Principais (Tab.3).

A representação plana das variáveis fenológicas e meteorológicas permitiu a definição de 3 grupos (Figura 2). Do grupo 1 participam as variáveis precipitação, "cacho com frutos maduros" e "palmeira sem cacho", que estão 


\section{ACTA AMAZONICA}

COMPORTAMENTO FENOLÓGICO DA PALMEIRA PATAUÁ (Oenocarpus bataua) NA RESERVA FLORESTAL ADOLPHO DUCKE, MANAUS, AMAZONAS, BRASIL
Tabela 3 - Contribuição percentual das variáveis fenológicas da palmeira Patauá (Oenocarpus bataua Martius) e meteorológicas nos componentes principais 1 e 2, no período de agosto de 1978 a dezembro de 1994 na Reserva Florestal Adolpho Ducke, Manaus, Amazonas, Brasil.

\begin{tabular}{lcc}
\hline \hline Variáveis & 1 & 2 \\
\hline CBF & 0,013 & 0,015 \\
CBE & 0,004 & 0,018 \\
CFA & $-0,002$ & 0,036 \\
CFC & $-0,007$ & 0,030 \\
CFCA & $-0,002$ & 0,025 \\
CFV & 0,003 & 0,023 \\
CFM & $-0,000$ & 0,067 \\
CFFC & $-0,038$ & 0,102 \\
CFCC & $-0,026$ & 0,126 \\
INS & $-0,315$ & 0,923 \\
PEM & 0,948 & 0,314 \\
TAM & $-0,006$ & 0,012 \\
TEM & $-0,002$ & 0,001 \\
TIM & 0,001 & $-0,002$ \\
URM & 0,018 & $-0,042$ \\
\hline Variação (\%) & 81,3 & 8,3 \\
\hline \hline
\end{tabular}

relacionadas à estação chuvosa. No grupo G3 participam as variáveis insolação, temperatura máxima e temperatura média e as fenofases, "cacho com frutos caindo" e "caídos" e "cacho com flores caindo" e "caídas", as quais estão ligadas à estação seca. No grupo G2 estão representadas as variáveis temperatura mínima e umidade relativa e as fenofases "cacho com bainha fechada","cacho com botões expostos", "cacho com flores abertas" e "cacho com frutos verdes", as quais têm um valor discriminatório intermediário. A temperatura mínima por estar situada na origem dos eixos tem representação nula, e a variação média anual da umidade relativa é praticamente constante.

\section{CONCLUSÕES}

As fenofases do cacho de patauá apresentaram-se em porcentagens $<14 \%$, com ocorrência o ano todo.

A precipitação e a insolação foram as variáveis que apresentaram alto poder discriminatório na formação dos componentes principais. As fenofases "cacho com frutos caindo" e "cacho com frutos caídos" apresentaram correlações positivas significativas somente em relação às variáveis insolação e temperatura média e máxima, e correlações negativas significativas com a precipitação e umidade relativa, com exceção de "cachos com frutos caindo" (correlação não significativa com a temperatura média), e "cachos com frutos caídos" (correlação significativa positiva com a umidade relativa).

As variáveis precipitação, "cachos com frutos maduros"

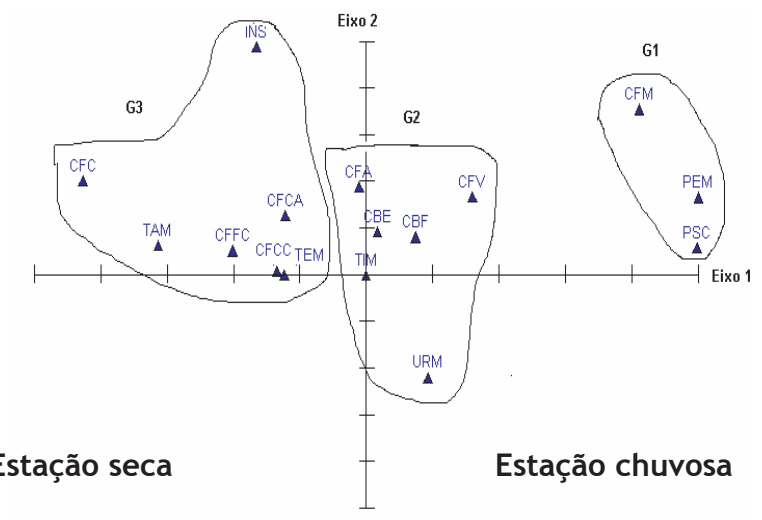

INS = insolação, PEM = precipitação, TAM = temperatura máxima, TEM = temperatura média; TIM = temperatura mínima; U RM = umidade relativa; $\mathrm{CBF}=$ cacho com bainha fechada; $\mathrm{CBE}=$ cacho com botões expostos; $C F A=$ cacho com flores abertas; $C F C=$ cacho com flores caindo; CFCA = cacho com flores caídas; $C F V=$ cacho com frutos verdes; CFM = cacho com frutos maduros; CFFC = cacho com frutos caindo; CFCC = cacho com frutos caídos; PSC = palmeira sem cacho.

Figura 2 - Representação plana das variáveis fenológicas de patauá (Oenocarpus bataua Martius) e meteorológicas no período de 1978 a dezembro de 1994, nos eixos 1 e 2 definidos pela análise dos componentes principais na Reserva Florestal Adolpho Ducke, Manaus, Amazonas, Brasil.

e "palmeira sem cacho" (que se relacionam com a estação chuvosa) estão em oposição às variáveis insolação, temperatura máxima, temperatura média e às fenofases "cacho com flores caindo" e "caídas" e "cacho com frutos caindo" e "caídos", as quais estão ligadas à estação seca.

\section{BIBLIOGRAFIA CITADA}

Alencar, J. C. 1994. Fenologia de cinco espécies arbóreas tropicais de Sapotaceae correlacionada a variáveis climáticas na Reserva Ducke, Manaus - Am. Acta Amazonica, 24(3/4):161-182.

Alencar, J. C.; Anderson, A. B. 1978. Projeto de pesquisa sobre 21 espécies de palmeiras na Reserva Florestal Adolpho Ducke. Departamento de Silvicultura Tropical - Instituto Nacional de Pesquisas da Amazônia. Manaus. 3p. Mimeografado. Inédito.

Araújo, V. C. 1970. Fenologia de essências florestais Amazônicas. I - Boletim do INPA, 4:25p.

Balick, M. J. 1992. Jessenia y Oenocarpus: palmas aceiteras neotropicales dignas de ser domesticadas. FAO, Estudio para la Producción y Protección Vegetal 88. Roma. 180p.

Balick, M.; Anderson, A. B. 1986/87. Dry matter allocation in Jessenia bataua (Palmae). Acta Amazonica, 16/17:135-140.

Bouroche, J. M.; Saporta, G. 1980. L'analyse des données. Que sais-je? Presses Universitaires de France,Paris. 127p.

Bowers, J. E.; Dimmitt, M. A. 1994. Flowering phenology of six woody plants in the northern Sonoran Desert. Bulletin of the Torrey Botanical Club, 121(3):215-229.

Bullock, S. H.; Solis-Magallanes, A. 1990. Phenology of canopy trees of a tropical deciduous forest in Mexico. Biotropica, 22(1):22-35. 


\section{ACTA AMAZONICA}

COMPORTAMENTO FENOLÓGICO DA PALMEIRA PATAUÁ (Oenocarpus bataua) NA RESERVA FLORESTAL ADOLPHO DUCKE, MANAUS, AMAZONAS, BRASIL
Collazos, M. E.; M. Mejia.1987. Fenologia y Postcosecha de Mil pesos, Jessenia bataua (Mart.) Burret. Tesis para Ingeniero Agronomo, Universidad Nacional de Colombia, Facultad de Ciencias Agropecuarias, Palmira, 120 p.

Ferraz, J.; Otha, S.; de Sales, P. C. 1998. Distribuição dos solos ao longo de dois transectos em floresta primária ao norte de Manaus (AM). In: Higuchi, N.; Campos, M. A. A.; Sampaio, P. T. B.; Santos, J. dos (Eds.). Pesquisas florestais para a conservação da floresta e reabilitação de áreas degradadas da Amazônia. Instituto Nacional de Pesquisas da Amazônia (INPA), Manaus, Amazonas. p.110-143.

García, M. 1988. Observaciones de polinización en Jessenia bataua (Arecaceae). Departamento de Ciencias Biológicas, Pontificia Universidad Católica del Ecuador, Quito. 60p.

Henderson, A.; Scariot, A. 1993. A flórula da Reserva Ducke, I: Palmae (Arecaceae). Acta Amazonica, 23(4):349-369.

Henderson, A. 1995. The Palms of the Amazon. Oxford University Press, New York. 361p.

Henderson, A.; Galeano, G. ; Bernal, R. 1995. Field Guide to the Palms of The Americas. Princeton University Press, Princeton, New Jersey, USA. 352p.

Ibarra-Manriquez, G. 1992. Fenologia de las palmas de uma selva cálido húmeda de Mexico. Bull. Inst. Fr. Études andines, 21(2):669-683.

Kahn, F. 1990. Las palmeras del arboretum Jenaro Herrera (Provincia de Requena, Departamento de Loreto, Perú). Contribución al estudio de la flora y de la vegetación de la Amazonía peruana. XVII. Candollea, 45: 341-362.

Kahn, F.; De Granville, J. J. 1992. Palms in Forest Ecosystems of Amazonia. Springer-Verlang, Berlin. 220p.

Neave, H. R. 1980. Tabelas Estatísticas para matemáticos, engenbeiros, economistas e profissionais administrativos e do comportamento. Ed. Difel, São Paulo. 88p.

Pedersen, H. B.; Balslev, H. 1993. Palmas Utiles, Especies Ecuatorianas para Agroforesteria y Extractivismo. Abya - Yala, Quito, Ecuador. 58p.

Peters, C. M.; Balick, M. J.; Kahn, F.; Anderson, A. B. 1989. Oligarchic forests of economic plants in Amazonia: utilization and conservation of an important tropical resource. Conservation Biology, 3(4): 341- 349 .
Phileppeau, G. 1992. Comment interpréter les résultats d'une analyse en composantes principales? Institut Technique des Céréales et des Fourrages, Paris. 63p

Ribeiro, J. E. L. da S.; Hopkins, M. J. G.; Vicentini, A.; Sothers, C. A.; Costa, M. A. da S.; Brito, J. M. de; Souza, M. A. D. de; Martins, L. H. P.; Lohmann, L. G.; Assunção, P. A. C. L.; Pereira, E. da C.; Silva, C. F. da; Mesquita, M. R.; Procópio, L. C. 1999. Flora da Reserva Ducke, guia de identificação das plantas vasculares de uma floresta de terra-firme na Amazônia Central.INPA,Manaus. 780p.

Rodriguez, J. C. 1995. Aspectos fitossociológicos das comunidades vegetais de uma toposseqüência da Reserva Florestal Ducke do INPA, Manaus - AM. Tese de Doutorado. Instituto Nacional de Pesquisas da Amazônia/Fundação Universidade do Amazonas, Manaus, Amazonas. 284p.

Ruiz, R. R. 1985. Ensayos de germinación com semillas de 5 espécies de palmeras aplicando 10 tratamientos pregerminativos y ensayos de cosecha con 7 métodos. Tesis para Ingeniero Forestal, Universidad Nacional de la Amazonia Peruana, Iquitos, Peru. 110p.

Sist, P. 1989 a. Peuplement et phénologie des palmiers en forêt guyanaise (Piste de Saint Elie). Rev. Ecolo. (Terre et Vie), 44:113-151.

Sist, P. 1989 b. Strategies de regeneration de quelques palmiers en forêt Guyanaise (Phénologie, Dissémination, Démographie). These de Doctorat de L'Université de Paris 6, Paris. 297p.

Wessel Boers, J. G. 1965. The indigenous palms of Suriname. E. J. Brill, Leiden. 172p.

Wood, P. J.; Burley, J. 1991. A tree for all reasons: Introduction and evaluation of multipurpose trees for agroforestry. Science and Practice of Agroforestry; ICRAF, Nairobi, Kenya. 158p

RECEBIDO EM: 18/02/2002 ACEITO EM: 21/10/2004 